studies, twin studies, and a high rate of positive family histories in case studies. The risk of PNE to parents is 6.7 -fold, and the risk to siblings 3.6-fold. Rates of enuresis in individuals with ADHD range from 20-30\%, compared to $10 \%$ in the average population of $7 \mathrm{yr}$ old children. In both conditions, boys are affected more frequently than girls. The frequency of ADHD is increased in PNE children compared to non-PNE controls. The present study suggests that the association of PNE and ADHD is not due to a shared genetic basis.

Factors in etiology of PNE other than genetic transmission are also considered in a commentary by Jarvelin M-R (Acta Paediatr Dec 1999;88:13151317). These include delayed maturation of the nervous system, disturbed arginine-vasopressin secretion, prenatal and birth-related factors, neurobehavioral disorders, and psychosocial factors. Many of these factors also apply to the presumed etiology of ADHD.

\title{
META-ANALYSIS STUDY OF CLONIDINE IN ADHD
}

The literature on the clinical use of clonidine for attention deficit hyperactivity disorder (ADHD), from 1980-1999, was reviewed at the Department of Psychiatry, University of Massachusetts Medical School, Worcester, MA. In 11 studies analyzed clonidine had positive effects in the treatment of ADHD and ADHD comorbid with conduct disorder, developmental delay, and tic disorder. The benefits were reported by clinician, parent, and teacher, and the effect was of moderate size $(0.58+/-0.16)$ and less than that of stimulants. The effect was significantly greater for the ADHD-alone group. Clonidine was associated with a high prevalence of side effects, especially sedation, irritability, night awakening, hypotension, and dizziness. Two studies using the transdermal system reported a high percentage of localized rash, erythema, and skin irritation under the patch. In one of 3 studies with ECG data, changes were noted but no cardiac symptoms. (Connor DF, Fletcher KE, Swanson JM. A meta-analysis of clonidine for symptoms of attention-deficit hyperactivity disorder. I Am Acad Child Adolesc Psychiatry Dec 1999;38:1551-1559). (Reprints: Dr Daniel F Connor, Department of Psychiatry, Room S7828 , University of Massachusetts Medical School, Worcester, MA 01655).

COMMENT. Clonidine is a second line treatment for ADHD and is less effective than stimulants, even in patients with comorbid disorders. A moderate size benefit is associated with a high incidence of side effects. In combination with stimulants, the risk of serious side effects is a concern, and a treatment to be avoided pending further study. The disappointing results of this meta-analysis may promote a greater interest in Tenex as an alternative treatment for ADHD children with comorbidity, including tics, insomnia, and oppositional defiance disorder.

\section{PANDAS, TICS AND OCD, AND IMMUNOTHERAPY}

Thirty children with severe, streptococcal-triggered exacerbations of tics or obsessive compulsive disorder $(O C D)$ were treated with plasma exchange (5 in 2 weeks), intravenous immunoglobulin (IVIG, $1 \mathrm{~g} / \mathrm{kg}$ daily on 2 consecutive days). or placebo saline solution, randomly assigned, in a study at the National Institutes of Health, Bethesda, MD. Neuropsychiatric medications were continued through the study, and oral penicillin or erythromycin was given during follow-up to protect against streptococcal infections. Throat cultures were negative at baseline, and antistreptolysin-O titers were positive in at least half the patients in each treatment group (mean 350-517). Similar elevations were found for AS deoxyribonucleic B titers. At 1 month, the improvements in the IVIG and plasma exchange groups were $45-58 \%$ for $O C D, 31-47 \%$ for anxiety, 33-35\% for overall 
functioning, and $49 \%$ on Tourette syndrome rating scale. The improvements following therapy were maintained at 1 year follow-up, with 14 (82\%) of 17 children benefited. (Perlmutter SJ, Leitman SF, Garvey MA et al. Therapeutic plasma exchange and intravenous immunoglobulin for obsessive-compulsive disorder and tic disorders in childhood. Lancet Oct 2, 1999;354:1153-1158). (Respond: Dr Susan E Swedo, 10 Center Drive-MSC 1255, Bethesda, MD 20892).

COMMENT. Immunotherapy with plasma exchange or immunoglobulin are successful in lessening severity of infection-triggered symptoms of obsessive compulsive disorder and Tourette syndrome. These positive results have only been demonstrated in a proportion of children with tics or OCD associated with PANDAS (post-infectious autoimmune neuropsychiatric disorders associated with streptococcal infection). The effects of immunotherapy in patients with chronic symptoms unrelated to PANDAS remains to be determined.

Singer HS in a commentary (Lancet Oct 1999;354: 1137-1138) notes that immunotherapies are not free of risk, two thirds having side effects. The sustained benefits reported might be explained by spontaneous improvements over time, especially for tics. Furthermore, medications had to be continued in at least half the patients.

An uncontrolled pilot trial in a small group of children with tics associated with ADHD and elevated ASO titers, oral penicillin treatment for 2 to 3 weeks has been associated with a decrease in tics (personal observation). The penicillin administered to all patients in the NIH study may have had a beneficial effect.

Abnormal cortical excitability in OCD and Tourette syndrome. Transcranial magnetic stimulation, previously showing decreased neuronal inhibition in the primary motor area of patients with Tourette syndrome, has demonstrated similar findings in $16 \mathrm{OCD}$ patients in a study at the $\mathrm{NIH}$, Bethesda, MD (Greenberg BD, Ziemann U, Cora-Locatelli G et al. Altered cortical excitability in obsessive-compulsive disorder. Neurology Jan 2000;54:142-147). The decreases in intracortical inhibition and motor threshold were greatest in OCD patients with comorbid tics.

Suprasellar germinoma presenting with Obsessive-Compulsive symptoms. A 13-year-old boy with a suprasellar germinoma involving the basal ganglia presented with psychotic and obsessive-compulsive symptoms in addition to hemiparesis, diabetes insipidus and impaired academic function (Mordecal D, Shaw RJ, Fisher PG et al. I Am Acad Child Adolesc Psychiatry Jan 2000;39:116-119). Other neurobehavioral disorders with basal ganglia involvement include Tourette syndrome and PANDAS.

\section{SEIZURE DISORDERS}

\section{VALPROATE-INDUCED ENCEPHALOPATHY}

Two adults, aged 32 and 37 , with focal epilepsy developed hyperammonemic encephalopathy when treated with a combination of valproate (VPA) and topiramate (TPM) at the University of Marburg, Germany. Previously the patients had tolerated combinations of VPA with phenobarbital or carbamazepine. Recovery followed the withdrawal of either VPA or TPM. (Hamer HM, Knake S, Schomburg U, Rosenow F. Valproate-induced hyperammonemic encephalopathy in the presence of topiramate. Neurology Jan 2000;54:230-232). (Reprints: Dr HM Hamer, Department of Neurology, University of Marburg, Rudolf-Bultmann-Str 8, 35033 